\title{
2573. Vibration stimulation effect on biomechanical variation of lower-limb joint according to vibration perception threshold
}

\author{
Kiyoung Kwak ${ }^{1}$, Huigyun Kim², Kyukeun Song ${ }^{3}$, Dongwook Kim ${ }^{4}$ \\ ${ }^{1,2}$ Department of Healthcare Engineering, Graduate School, Chonbuk National University, \\ Jeonju, Republic of Korea \\ ${ }^{3}$ Department of Mechanical System Engineering, College of Engineering, Chonbuk National University, \\ Jeonju, Republic of Korea \\ ${ }^{4}$ Department of Biomedical Engineering, College of Engineering, Chonbuk National University, \\ Jeonju, Republic of Korea \\ ${ }^{4}$ Research Center of Healthcare and Welfare Instrument for Aged, Chonbuk National University, \\ Jeonju, Republic of Korea \\ ${ }^{4}$ Corresponding author \\ E-mail: ${ }^{1} k y k w a k 86 @ n a t e . c o m,{ }^{2} h e k u n 1249 @ n a v e r . c o m,{ }^{3}$ songkk@jbnu.ac.kr, ${ }^{4}$ biomed@jbnu.ac.kr
}

Received 15 May 2017; received in revised form 5 July 2017; accepted 24 July 2017

DOI https://doi.org/10.21595/jve.2017.18886

Check for updates

\begin{abstract}
Vibration applied to relaxed muscle or tendon causes tonic reflex contraction in a muscle. Furthermore, when vibration is applied to a muscle, involuntary enhancement of EMG and contraction is induced. Regarding this finding, many studies on the influence of vibration on a muscle and static posture have been performed. However, precedent studies have limitations in applying single characteristic-vibration without any consideration on personal difference on vibration. And studies have been performed to research the change in dynamic state according to the intensity of sensing vibration, but no analysis has been performed on the biomechanical aspect of the lower-limb joints. Nor any consideration was given on the effect of vibration frequency. Therefore, the purpose of this study was to analyze the biomechanical variation in the lower-limb joints according to the characteristics of the mechanical vibration stimulation flowing into Achilles tendon and tibialis anterior tendon during gait, in consideration of the vibration perception threshold and vibration frequency. For this purpose, this study measures the vibration perception threshold according to vibration frequency at each tendon exposed to the stimulation. According to the result, vibration perception threshold varies according to vibrating tendon and vibration frequency. Based on the measurement result of vibration perception threshold, vibration is applied to an Achilles tendon and tibialis anterior tendon during gait. In order to analyze the biomechanical variation in the lower-limb joints according to the characteristics of vibration stimulation applied to each tendon during gait, the angle, moment and power of the lower-limb joints is analyzed using 3D motion analysis system. As a result, biomechanical variation, when vibration lower than a perception threshold is applied, is similar to the variation when vibration at perception threshold is applied. This result implies that vibration stimulation may cause biomechanical variation of lower-limb joints. Furthermore, this means that its biomechanical variation may vary according to the characteristics of the vibration applied.
\end{abstract}

Keywords: somatosensory, vibration, biomechanics, gait, perception threshold, postural control.

\section{Introduction}

Sustained vibration applied to initially relaxed skeletal muscle or its tendon induces tonic reflex contraction in a muscle [1]. Moreover, when vibration is applied to a muscle engaged in a weak or moderate voluntary contraction, involuntary enhancement of electromyogram (EMG) and contraction strength is induced $[2,3]$. Based on this finding, a number of studies on the influence of vibration on a muscle have been performed.

Burke et al. researched what response occurs when vibration is applied to muscle in non-contracting, isometric contraction condition $[4,5]$. Bongiovanni et al. found that EMG 
develops when vibration is applied to a muscle in initially relaxed condition, and also that EMG and Force develops as vibration is applied when constant effort is maintained [6]. And it is also found that EMG and Force increases momentarily and then abruptly decreases when vibration is applied during maximal voluntary contraction (MVC) of muscle in a fatigued condition, and EMG and Force shows post-vibratory recovery again when inpouring of vibration is stopped [6]. Based on this result, they revealed that prolonged muscle vibration reduces MVC output [7]. As such, a number of studies have been performed on the influence of vibration on the muscle.

There are many studies on the effect of vibration on posture within a different category from muscles. Eklund et al. reveals, based on their study, that the muscle activation induced by vibration influences postural stability [8]. Subsequently, many studies have been performed on the influence of vibration on posture. Recently applying them, studies have been performed reporting that postural stability is increased or postural sway is reduced when vibration is applied to upright posture, stable posture, and unstable posture [9-12]. As is the case on the studies on postural stability, many studies are reported by applying to muscle performance [12]. Among the related studies, Curry et al. demonstrates that maximal voluntary contraction of muscle increases when vibration stimulation is applied. [14]. Kihlberg et al. demonstrates that intergraded EMG (IEMG) increases [15]. Based on these studies, vibration is often applied to physical training [16]. However, precedent studies have limitations in applying single characteristic-vibration without any consideration on personal difference on vibration. In other words, precedent studies failed to reflect the sensitivity to vibration, i.e. the degree of sensing vibration. Thereafter, studies have been performed to research the change in dynamic state according to the intensity of sensing vibration $[17,18]$, but no analysis has been performed on the biomechanical aspect of the lower-limb. Nor any consideration was given on the effect of vibration frequency. Therefore, the purpose of this study is to analyze the biomechanical variation in the lower-limb according to the characteristics of vibration stimulation applied during gait, in consideration of vibration perception threshold and vibration frequency.

\section{Methods}

\subsection{Subjects}

A total of 15 healthy subjects (Age: 26.4 \pm 1.5 , Height: 171.6 \pm 2.7 Weight: 66.1 \pm 5.7 ) participated in this study. All subjects to the experiment have no diseases in their nervous system and musculoskeletal system, capable of gait independently without any assisting device. This study was approved by the IRB of Chonbuk National University (IRB File No. JBNU 2015-06-012).

\subsection{Equipment}

A Linear actuator (0934, Samsung electro-mechanics, Korea) was used to apply the vibration stimulation on the Achilles tendon and the tibialis anterior tendon. Function generator (8202-2 MHz Generator, Dagatron corp., Korea) was used to moderate the frequency and intensity of the vibration to be applied on these tendons.

To capture the movement in gait, a total of 21 active infrared emitting diode markers were attached to each major joint part according to a whole-body marker set. To collect the infrared light from the markers, a total of 3 Position Sensors (Optotrak Certus, Northern Digital Inc, Canada) were used. To measure the ground reaction force during gait, a total of 4 force plates (Bertec Co., Ltd, U.S.A) were used.

\subsection{Vibration application}

The Achilles tendon (ACT) and Tibialis anterior tendon (TAT) which could affect the 
lower-limb muscle through vibration stimulation, were selected as the targeted part for applying. To investigate the biomechanical variation in the lower-limb according to the characteristics of the applied vibration, intensity of vibration was adjusted and vibration perception threshold was measured on the vibration frequency per each tendon exposed to stimulation (Fig. 1). Based on measurement result (Fig. 1) and previously study [19], vibration is applied to tendon during gait.

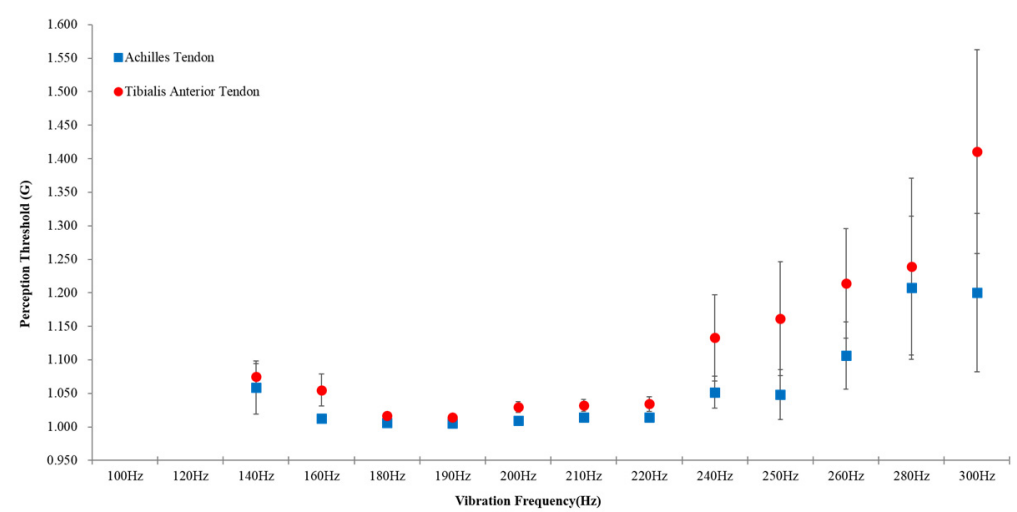

Fig. 1. Vibration perception threshold on Achilles and tibialis anterior tendon (No anyone felt $100 \mathrm{~Hz}$ and $120 \mathrm{~Hz}$ frequency)

\subsection{Vibration stimulation condition}

By combining the stimulating part of vibration inpouring and vibration perception threshold, vibration condition is set. In case of no vibration applied, non-stimulation appeared. In case of vibration at perception threshold level (threshold vibration) is applied to Achilles tendon, ACT_100\% appeared. In case vibration at $80 \%$ of perception threshold (sub-threshold) is applied to Achilles tendon, ACT_80\% appeared. In case of applying to tibialis anterior tendon, TAT_100\% and TAT_ $80 \%$ appeared, respectively. Thus, a total of 13 different vibration stimulation condition were set.

\subsection{Protocol}

The subjects walked at least $10 \mathrm{~m}$ at self-selected speed. The vibration stimulation were randomly applied. The subjects performed gaits 3 times per each stimulation condition. And, they take a break of 3 minutes after finishing each vibration stimulation condition.

\subsection{Analysis}

To investigate the biomechanical variation in the lower-limb according to the characteristics of vibration applied during gait, a 3-dimensional human musculoskeletal system modeling and analysis software (SIMM, MusculoGraphics Inc., USA) was used. The stance phase during gait was set as the range for analysis. The angle, moment and power of a lower-limb during a stance phase were taken as the analysis parameter. Using the mean angle, moment and power of each joint at three gaits on flat ground, the mean variation at each vibration stimulation condition are presented as the result. Using this, comparative analysis was performed on the comparison between average variations in non-stimulation condition and vibration stimulation conditions, the comparison in average variation according to vibration perception threshold. 


\section{Results}

\subsection{Ankle joint biomechanics according to vibration perception threshold}

The angle, moment and power of ankle joint are analyzed according to vibration perception threshold, and the result was presented by each part exposed to stimulation (Table 1-2).

When an Achilles tendon is exposed to stimulation, the dorsiflexion increased more than in condition of non-stimulation, at all perception threshold intensity and perception sub-threshold intensity condition (Table 1). Similarly, when tibialis anterior tendon is exposed to stimulation, the dorsiflexion increased more than in condition of non-stimulation, at all perception threshold intensity and perception sub-threshold intensity condition (Table 2). There are increased and decreased pattern in moment. And positive power decreased more than non-stimulation condition, at all vibration stimulation condition.

Table 1. Ankle joint biomechanical variation according to vibration perception threshold in ACT stimulation ACT: Achilles Tendon, TAT: Tibialis Anterior Tendon

\begin{tabular}{|c|c|c|c|c|}
\hline \multicolumn{2}{|c|}{ Ankle joint } & Non-stimulation & ACT $100 \%$ & ACT $80 \%$ \\
\hline \multirow{3}{*}{$180 \mathrm{~Hz}$} & Angle & $12.13 \pm 3.44$ & $12.44 \pm 3.48$ & $12.72 \pm 3.38$ \\
\cline { 2 - 5 } & Moment & $-0.718 \pm 0.229$ & $-0.719 \pm 0.232$ & $-0.730 \pm 0.231$ \\
\cline { 2 - 5 } & Power & $0.132 \pm 0.497$ & $0.106 \pm 0.498$ & $0.130 \pm 0.489$ \\
\hline \multirow{3}{*}{$190 \mathrm{~Hz}$} & Angle & $12.13 \pm 3.44$ & $12.26 \pm 3.36$ & $12.67 \pm 3.36$ \\
\cline { 2 - 5 } & Moment & $-0.718 \pm 0.229$ & $-0.717 \pm 0.228$ & $-0.711 \pm 0.228$ \\
\cline { 2 - 5 } & Power & $0.132 \pm 0.497$ & $0.142 \pm 0.493$ & $0.112 \pm 0.482$ \\
\hline \multirow{3}{*}{$250 \mathrm{~Hz}$} & Angle & $12.13 \pm 3.44$ & $12.32 \pm 3.26$ & $12.29 \pm 3.53$ \\
\cline { 2 - 5 } & Moment & $-0.718 \pm 0.229$ & $-0.727 \pm 0.226$ & $-0.708 \pm 0.235$ \\
\cline { 2 - 5 } & Power & $0.132 \pm 0.497$ & $0.115 \pm 0.486$ & $0.117 \pm 0.508$ \\
\hline
\end{tabular}

Table 2. Ankle joint biomechanical variation according to vibration perception threshold in TAT stimulation ACT: Achilles Tendon, TAT: Tibialis Anterior Tendon

\begin{tabular}{|c|c|c|c|c|}
\hline \multicolumn{2}{|c|}{ Ankle joint } & Non-stimulation & TAT $100 \%$ & TAT $80 \%$ \\
\hline \multirow{3}{*}{$180 \mathrm{~Hz}$} & Angle & $11.87 \pm 3.54$ & $12.36 \pm 3.65$ & $12.53 \pm 3.62$ \\
\cline { 2 - 5 } & Moment & $-0.718 \pm 0.229$ & $-0.731 \pm 0.231$ & $-0.718 \pm 0.234$ \\
\cline { 2 - 5 } & Power & $0.132 \pm 0.497$ & $0.131 \pm 0.531$ & $0.100 \pm 0.497$ \\
\hline \multirow{3}{*}{$190 \mathrm{~Hz}$} & Angle & $11.87 \pm 3.54$ & $12.14 \pm 3.61$ & $12.24 \pm 3.55$ \\
\cline { 2 - 5 } & Moment & $-0.718 \pm 0.229$ & $-0.716 \pm 0.230$ & $-0.724 \pm 0.228$ \\
\cline { 2 - 5 } & Power & $0.132 \pm 0.497$ & $0.105 \pm 0.500$ & $0.113 \pm 0.474$ \\
\hline \multirow{3}{*}{$250 \mathrm{~Hz}$} & Angle & $11.87 \pm 3.54$ & $12.15 \pm 3.63$ & $12.13 \pm 3.61$ \\
\cline { 2 - 5 } & Moment & $-0.718 \pm 0.229$ & $-0.721 \pm 0.228$ & $-0.711 \pm 0.232$ \\
\cline { 2 - 5 } & Power & $0.132 \pm 0.497$ & $0.112 \pm 0.505$ & $0.127 \pm 0.496$ \\
\hline
\end{tabular}

In condition of vibration stimulation on the Achilles and tibialis anterior tendon, the dorsiflexion increase when threshold intensity vibration was applied. This tendency appears at sub-threshold intensity vibration stimulation condition as well. The center of mass (COM) was lowered due to increased dorsiflexion during the stance phase, and this would contribute to the body stability during gait. The movement of the COM is the variable that actually causes the sway of the whole body [20]. The maintenance of stability during gait is dependent on the ability to control COM motion [21]. High stability is characterized by a large base of support, a low center of mass, a centralized center of gravity projection within the base of support, a large body mass [22]. When threshold intensity vibration was applied, positive power decreased in condition of stimulation on the Achilles and tibialis anterior tendon. And this result, appears at perception sub-threshold intensity vibration stimulation condition as well. This means that gradually increase of negative power by plantar flexor. Through this result, it can be assumed that vibration contributed to the absorption of shocks from the ground, support of human body and enhanced stabilization of the joints. Therefore, vibration is found as contributing positively to the stability 
of ankle joints during gait. And as this tendency appears at sub-threshold, sub-threshold vibration may be assumed as contributing to the stability and function of ankle joints.

Consistent patterns are disappeared according to vibration stimulation in moment of stimulation on tibialis anterior. But, peak of plantar flexor moment increased than non-stimulation condition (Table 3). Thus, tibialis anterior vibration stimulation would contribute to increase of support-moment.

A ankle extensor moment (plantar flexor moment), for example, means that the ankle extensors (soleus, gastrocnemius) are dominant at the ankle joint and the ankle extensors are creating a greater moment than the ankle flexors (tibialis anterior). Collapse of the lower-limb requires a flexion at all three joints (knee, ankle and hip), thus support of the body requires net extensor activity at these joints [23]. The support-moment is the sum of the extensor moments of the three joints of the lower-limb [24]. The sum of the extensor muscles moments of the lower-limb joints must be positive to support the body [25]. When the support-moment is positive, it supports the lower-limbs and prevents their collapse [23]. It also means that when on joint opposes or does not contribute to lower limb support, one or both of the other joints will compensate or the non-contributing joints [23]. Therefore, tibialis anterior stimulation of perception threshold intensity and perception sub-threshold intensity would contribute to lower-limb support and prevents their collapse. Although plantar flexor moment decreased in $190 \mathrm{~Hz}$ and $250 \mathrm{~Hz} 80 \%$ \% of Achilles tendon stimulation, knee and hip joints will compensate plantar flexor moment.

Table 3. Ankle peak plantar flexor moment biomechanical variation according to vibration perception threshold in TAT stimulation ACT: Achilles Tendon, TAT: Tibialis Anterior Tendon

\begin{tabular}{|c|c|c|c|c|}
\hline \multicolumn{2}{|c|}{ Ankle joint } & Non-stimulation & TAT $100 \%$ & TAT $80 \%$ \\
\hline \multirow{2nnny}{*}{$180 \mathrm{~Hz}$} & \multirow{3}{*}{ Peak plantar flexor moment } & \multirow{2}{*}{-1.397} & -1.424 & -1.416 \\
\cline { 1 - 3 } & & & -1.406 & -1.400 \\
\hline $250 \mathrm{~Hz}$ & & -1.402 & -1.410 \\
\hline
\end{tabular}

\subsection{Knee joint biomechanics according to vibration perception threshold}

The angle, moment and power of knee joint are analyzed according to vibration perception threshold, and the result was presented by each part exposed to stimulation (Table 4-5).

Table 4. Knee joint biomechanical variation according to vibration perception threshold in ACT stimulation ACT: Achilles Tendon, TAT: Tibialis Anterior Tendon

\begin{tabular}{|c|c|c|c|c|}
\hline \multicolumn{2}{|c|}{ Knee Joint } & Non-stimulation & ACT $100 \%$ & ACT $80 \%$ \\
\hline \multirow{3}{*}{$180 \mathrm{~Hz}$} & Angle & $17.50 \pm 3.72$ & $17.46 \pm 3.71$ & $17.68 \pm 3.69$ \\
\cline { 2 - 5 } & Moment & $-0.264 \pm 0.079$ & $-0.271 \pm 0.079$ & $-0.270 \pm 0.074$ \\
\cline { 2 - 5 } & Power & $-0.266 \pm 0.080$ & $-0.259 \pm 0.087$ & $-0.270 \pm 0.092$ \\
\hline \multirow{3}{*}{$190 \mathrm{~Hz}$} & Angle & $17.50 \pm 3.72$ & $17.67 \pm 3.73$ & $17.23 \pm 3.64$ \\
\cline { 2 - 5 } & Moment & $-0.264 \pm 0.079$ & $-0.271 \pm 0.079$ & $-0.266 \pm 0.074$ \\
\cline { 2 - 5 } & Power & $-0.266 \pm 0.080$ & $-0.282 \pm 0.091$ & $-0.252 \pm 0.081$ \\
\hline \multirow{3}{*}{$250 \mathrm{~Hz}$} & Angle & $17.50 \pm 3.72$ & $17.24 \pm 3.76$ & $17.61 \pm 3.80$ \\
\cline { 2 - 5 } & Moment & $-0.264 \pm 0.079$ & $-0.267 \pm 0.076$ & $-0.284 \pm 0.078$ \\
\cline { 2 - 5 } & Power & $-0.266 \pm 0.080$ & $-0.286 \pm 0.085$ & $-0.286 \pm 0.089$ \\
\hline
\end{tabular}

When an Achilles tendon is exposed to stimulation, an increase of flexion and negative power was more prevalent than in condition of non-stimulation, and knee extensor moment of all increased at all vibration stimulation condition (Table 4). When tibialis anterior tendon is exposed to stimulation, the flexion, extensor moment and negative power increased more than in condition of non-stimulation, at all perception threshold intensity and perception sub-threshold intensity vibration condition (Table 5).

When vibration stimulation of perception threshold intensity and sub-threshold intensity applied to tibialis anterior tendon, knee extensor moment and negative power increased more than 
in non-stimulation condition, at all perception threshold intensity and perception sub-threshold intensity vibration stimulation condition. This means that TAT vibration stimulation of perception threshold intensity and sub-threshold intensity will contribute to decrease of height of center of mass (COM) and to lower-limb support and prevents their collapse.

In ACT stimulation condition, extensor moment increased more than non-stimulation condition and increase of flexion and negative power appeared prevalent. It seems that ACT stimulation positively contribute to body support and prevent lower-limb collapse. And particularly increased knee extensor moment will compensate decreased ankle plantar flexor moment of ACT_190 Hz and $250 \mathrm{~Hz} \_80 \%$.

Table 5. Knee joint biomechanical variation according to vibration perception threshold in TAT stimulation ACT: Achilles Tendon, TAT: Tibialis Anterior Tendon

\begin{tabular}{|c|c|c|c|c|}
\hline \multicolumn{2}{|c|}{ Knee Joint } & Non-stimulation & TAT_100\% & TAT $80 \%$ \\
\hline \multirow{3}{*}{$180 \mathrm{~Hz}$} & Angle & $17.50 \pm 3.72$ & $18.21 \pm 3.74$ & $18.16 \pm 3.68$ \\
\cline { 2 - 5 } & Moment & $-0.264 \pm 0.079$ & $-0.267 \pm 0.081$ & $-0.277 \pm 0.080$ \\
\cline { 2 - 5 } & Power & $-0.266 \pm 0.080$ & $-0.282 \pm 0.091$ & $-0.312 \pm 0.086$ \\
\hline \multirow{3}{*}{$190 \mathrm{~Hz}$} & Angle & $17.50 \pm 3.72$ & $17.74 \pm 3.75$ & $17.59 \pm 3.65$ \\
\cline { 2 - 5 } & Moment & $-0.264 \pm 0.079$ & $-0.278 \pm 0.083$ & $-0.280 \pm 0.080$ \\
\cline { 2 - 5 } & Power & $-0.266 \pm 0.080$ & $-0.305 \pm 0.096$ & $-0.300 \pm 0.092$ \\
\hline \multirow{3}{*}{$250 \mathrm{~Hz}$} & Angle & $17.50 \pm 3.72$ & $17.58 \pm 3.77$ & $17.97 \pm 3.70$ \\
\cline { 2 - 5 } & Moment & $-0.264 \pm 0.079$ & $-0.279 \pm 0.083$ & $-0.271 \pm 0.077$ \\
\cline { 2 - 5 } & Power & $-0.266 \pm 0.080$ & $-0.283 \pm 0.089$ & $-0.275 \pm 0.081$ \\
\hline
\end{tabular}

\subsection{Hip joint biomechanics according to vibration perception threshold}

The angle, moment and power of hip joint are analyzed according to vibration perception threshold, and the result was presented by each part exposed to stimulation (Tables 6-7).

Table 6. Hip joint biomechanical variation according to vibration perception threshold in ACT stimulation ACT: Achilles Tendon, TAT: Tibialis Anterior Tendon

\begin{tabular}{|c|c|c|c|c|}
\hline \multicolumn{2}{|c|}{ Hip joint } & Non-stimulation & ACT $100 \%$ & ACT $80 \%$ \\
\hline \multirow{3}{*}{$180 \mathrm{~Hz}$} & Angle & $6.77 \pm 7.67$ & $6.92 \pm 7.46$ & $7.71 \pm 7.55$ \\
\cline { 2 - 5 } & Moment & $-0.112 \pm 0.192$ & $-0.115 \pm 0.183$ & $-0.130 \pm 0.190$ \\
\cline { 2 - 5 } & Power & $0.442 \pm 0.146$ & $0.406 \pm 0.136$ & $0.431 \pm 0.143$ \\
\hline \multirow{3}{*}{$190 \mathrm{~Hz}$} & Angle & $6.77 \pm 7.67$ & $7.33 \pm 7.70$ & $7.54 \pm 7.57$ \\
\cline { 2 - 5 } & Moment & $-0.112 \pm 0.192$ & $-0.130 \pm 0.191$ & $-0.148 \pm 0.191$ \\
\cline { 2 - 5 } & Power & $0.442 \pm 0.146$ & $0.446 \pm 0.139$ & $0.438 \pm 0.135$ \\
\hline \multirow{3}{*}{$250 \mathrm{~Hz}$} & Angle & $6.77 \pm 7.67$ & $7.45 \pm 7.58$ & $7.63 \pm 7.63$ \\
\cline { 2 - 5 } & Moment & $-0.112 \pm 0.192$ & $-0.123 \pm 0.191$ & $-0.127 \pm 0.194$ \\
\cline { 2 - 5 } & Power & $0.442 \pm 0.146$ & $0.429 \pm 0.136$ & $0.434 \pm 0.143$ \\
\hline
\end{tabular}

Table 7. Hip joint biomechanical variation according to vibration perception threshold in TAT stimulation ACT: Achilles Tendon, TAT: Tibialis Anterior Tendon

\begin{tabular}{|c|c|c|c|c|}
\hline \multicolumn{2}{|c|}{ Hip joint } & Non-stimulation & TAT $100 \%$ & TAT $80 \%$ \\
\hline \multirow{3}{*}{$180 \mathrm{~Hz}$} & Angle & $6.77 \pm 7.67$ & $7.89 \pm 7.66$ & $8.21 \pm 7.68$ \\
\cline { 2 - 5 } & Moment & $-0.112 \pm 0.192$ & $-0.140 \pm 0.193$ & $-0.165 \pm 0.191$ \\
\cline { 2 - 5 } & Power & $0.442 \pm 0.146$ & $0.446 \pm 0.140$ & $0.439 \pm 0.136$ \\
\hline \multirow{3}{*}{$190 \mathrm{~Hz}$} & Angle & $6.77 \pm 7.67$ & $7.97 \pm 7.70$ & $7.88 \pm 7.65$ \\
\cline { 2 - 5 } & Moment & $-0.112 \pm 0.192$ & $-129 \pm 0.186$ & $-0.132 \pm 0.192$ \\
\cline { 2 - 5 } & Power & $0.442 \pm 0.146$ & $0.432 \pm 0.138$ & $0.426 \pm 0.137$ \\
\hline \multirow{3}{*}{$250 \mathrm{~Hz}$} & Angle & $6.77 \pm 7.67$ & $7.90 \pm 7.58$ & $8.16 \pm 7.66$ \\
\cline { 2 - 5 } & Moment & $-0.112 \pm 0.192$ & $-0.122 \pm 0.189$ & $-0.148 \pm 0.189$ \\
\cline { 2 - 5 } & Power & $0.442 \pm 0.146$ & $0.413 \pm 0.136$ & $0.427 \pm 0.140$ \\
\hline
\end{tabular}


Flexion and Extensor moment increased in vibration stimulation condition of all. And decrease of positive power appeared prevalent (Tables 6-7). This result indicate that vibration stimulation of perception threshold intensity and sub-threshold intensity is contribute positively to hip joint function about stability during gait. Also, increased hip extensor moment will compensate decreased ankle plantar flexor moment of ACT_190 Hz and $250 \mathrm{~Hz} 80 \%$. And from decreased of positive power, it can be assumed that vibration contributed to the absorption of shocks from the ground, support of human body and enhanced stabilization of the joints.

\section{Conclusions}

This study investigated the biomechanical variation in the lower-limb according to vibration perception threshold during stance phase, and derives the conclusion as follows.

When vibration is applied to a tendon, the angle, moment and power of the lower-limb is affected to cause variation. And the variation that occurred at threshold vibration stimulation also occurred upon sub-threshold vibration stimulation as well. As a result, increase of flexion and extensor moment and decrease of positive power appeared prevalent in vibration stimulation condition.

Through this result, it can be assumed that vibration stimulation contributed to decrease of height of COM and to lower-limb support and prevents their collapse. Consequently, joint function about absorption of shocks from the ground, support of human body and stabilization of the joints are enhanced.

\section{Acknowledgements}

Research Foundation of Korea (NRF) Grant funded by the Korea Government (MSIP) (NRF-2014R1A2A1A11053073).

\section{References}

[1] Eklund G., Hagbarth K. E. Normal variability of tonic vibration reflexes in man. Experimental Neurology, Vol. 16, Issue 1, 1966, p. 80-92.

[2] Hagbarth K. E., Eklund G. Motor effects of vibratory muscle stimuli in man. Muscular Afferents and Motor Control, Almqvist and Wiksell, Stockholm, 1966, p. 177-186.

[3] Mccloskey D. J., Ebeling P., Goodwin G. M. Estimation of weights and tension and apparent involvement of a 'sense of effort'. Experimental Neurology, Vol. 42, Issue 1, 1974, p. 220-232.

[4] Burke D., Hagbarth K. E., Lofstedt L., Walling B. G. The response of human muscle spindle endings to vibration of non-contracting muscle. Journal of Physiology, Vol. 261, Issue 3, 1976, p. 673-693.

[5] Burke D., Hagbarth K. E., Lofstedt L., Walling B. G. The response of human muscle spindle endings to vibration during isometric contraction. Journal of Physiology, Vol. 261, Issue 3, 1976, p. 695-711.

[6] Bongiovanni L. G., Hagbarth K. E. Tonic vibration reflexes elicited during fatigue from maximal voluntary contractions in man. Journal of Physiology, Vol. 423, Issue 1, 1990, p.1-14.

[7] Bongiovanni L. G., Hagbarth K. E., Stjernberg L. Prolonged muscle vibration reducing motor output in maximal voluntary contractions in man. Journal of Physiology, Vol. 423, Issue 1, 1990, p. $15-26$.

[8] Eklund G. General features of vibration-induced effects on balance. Upsala Journal of Medical Sciences, Vol. 77, Issue 2, 1972, p. 112-124.

[9] Slijper H., Latash M. L. The effects of muscle vibration on anticipatory postural adjustments. Brain Research, Vol. 1015, Issues 1-2, 2004, p. 57-72.

[10] Ceyte H., Cian C., Zory R., Barraud P. A., Roux A., Guerraz M. Effect of Achilles tendon vibration on postural orientation. Neuroscience Letters, Vol. 416, Issue 1, 2007, p. 71-75.

[11] Thompson C., Belanger M., Fung J. Effects of bilateral Achilles tendon vibration on postural orientation and balance during standing. Clinical Neurophysiology, Vol. 118, Issue 11, 2007, p. 2456-2467. 
[12] Thompson C., Belanger M., Fung J. Effects of plantar cutaneo-muscular and tendon vibration on posture and balance during quiet and perturbed stance. Human Movement Science, Vol. 30, Issue 2, 2011, p. 153-171.

[13] Luo J., McNamara B., Moran K. The use of vibration training to enhance muscle strength and power. Sports Medicine, Vol. 35, Issue 1, 2005, p. 23-41.

[14] Curry E. L., Clelland J. A. Effects of the asymmetric tonic neck reflex and high-frequency muscle vibration on isometric wrist extension strength in normal adults. Physical Therapy, Vol. 61, Issue 4, 1981, p. 487-495.

[15] Kihlberg S., Attebrant M., Gemne G. Acute effects of vibration from a chipping hammer and a grinder on the hand-arm system. Occupational and Environmental Medicine, Vol. 52, Issue 11, 1995, p. 731-737.

[16] Luo J., McNamara B., Moran K. A portable vibrator for muscle performance enhancement by means of direct muscle tendon stimulation. Medical Engineering and Physics, Vol. 27, Issue 6, 2005, p. 513-522.

[17] So H. J., Kim S. H., Kim D. W. A study on the changes in gait characteristics by applying subthreshold vibration stimulus in the ankle. Journal of Vibroengineering, Vol. 16, Issue 7, 2014, p. 3331-3339.

[18] So H. J., Kim S. H., Kim D. W. The effects of application site and time of vibration stimulation: Changes in gait pattern and muscle activity. Applied Mechanics and Materials, Vols. 479-480, 2014, p. 475-479.

[19] Kwak K. Y, Kim H. G., Kim D. W. Variation of ankle biomechanical property according to vibroperception threshold and vibration frequency. Biomedical Engineering Letters, Vol. 6, Issue 1, 2016, p. $16-25$.

[20] Almoudi M., Stambolian D., Asfour S. Center of mass deviation from center of base of support as a measure of frontal and sagittal stability. International Journal of Biomedical Engineering and Science, Vol. 3, Issue 1, 2016, p. 1-18.

[21] Mian O. S., Narici M. V., Minetti A. E., Baltzopoulos V. Centre of mass motion during stair negotiation in young and older men. Gait and Posture, Vol. 26, Issue 3, 2007, p. 463-469.

[22] Whiting W. C., Rugg S. Dynatomy: Dynamic Human Anatomy. Human Kinetics Publishers, Champaign, 2012.

[23] Winter D. A. Overall principle of lower limb support during stance phase of gait. Journal of Biomechanics, Vol. 13, Issue 11, 1980, p. 923-927.

[24] Hof A. L. On the interpretation of the support moment. Gait and Posture, Vol. 12, Issue 3, 2000, p. 196-199.

[25] McNee A. E., Shortland A. P., Eve L. C., Robinson R. O., Gough M. Lower limb extensor moments in children with spastic diplegic cerebral palsy. Gait and Posture, Vol. 20, Issue 2, 2004, p. 171-176.

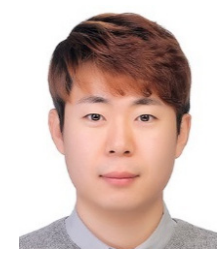

Kiyoung Kwak is now with the Department of Healthcare Engineering, Chonbuk National University, Jeonju-si, Jeollabuk-do, Republic of Korea. His current research interests include neuro-musculoskeletal biomechanics and somatosensory-motor integration.

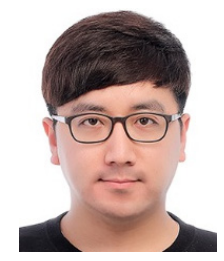

Huigyun Kim is now with the Department of Healthcare Engineering, Chonbuk National University, Jeonju-si, Jeollabuk-do, Republic of Korea. His current research interests include neuronal electrophysiology and sensory-motor integration. 


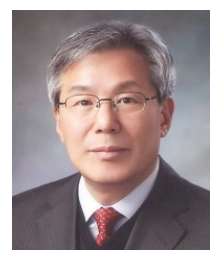

Kyukeun Song received Ph.D. degree in mechanical engineering from Hokkaido University, Sapporo, Japan in 1987. He was dean of the college of engineering from 2008 to 2010. Now he is a Professor at Department of Mechanical System Engineering, Chonbuk National University, Jeonju-si, Jeollabuk-do, Republic of Korea. His current research interests include biomedical engineering, mechanical system engineering.

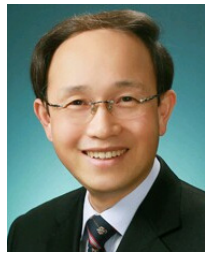

Dongwook Kim received Ph.D. degree in biomedical engineering from Hokkaido University, Sapporo, Japan in 1995. Now he is a Professor at Department of Biomedical Engineering and director of Keunsaram Educational Development Institute in Chonbuk National University, Jeonju-si, Jeollabuk-do, Republic of Korea. His current research interests include biomedical engineering, rehabilitation engineering, sensory-motor integration, diagnosis and healthcare system. 